\title{
The effect of prenatal exposure to 900-megahertz electromagnetic field on the newborn rat testicles
}

\author{
Hatice Hancı $^{a}$, Ersan Odacı* ${ }^{a *}$ Haydar Kaya ${ }^{\mathrm{b}}$, Yüksel Aliyazıcıoğluc ${ }^{\mathrm{i}}$ İbrahim Turan ${ }^{\mathrm{d}}$, Selim Demir $^{\mathrm{c}}$, Serdar Çolakoğlu \\ ${ }^{a}$ Department of Histology and Embryology, Faculty of Medicine, Karadeniz Technical University, Trabzon, Turkey \\ ${ }^{b}$ Department of Electrical and Electronics Engineering, Faculty of Engineering, Karadeniz Technical University, Trabzon, \\ Turkey \\ ${ }^{c}$ Department of Medical Biochemistry, Faculty of Medicine, Karadeniz Technical University, Trabzon, Turkey \\ ${ }^{d}$ Department of Genetic and Bioengineering, Faculty of Engineering, Gümüşane University, Gümüşane, Turkey \\ ${ }^{e}$ Department of Anatomy, Faculty of Medicine, Düzce University, Düzce, Turkey
}

\section{ARTICLE INFO \\ * Correspondence to: \\ Ersan Odac1 \\ Department of Histology and Embryology, Faculty of Medicine, \\ Karadeniz Technical University, \\ Trabzon, Turkey \\ e-mail: ersanodaci@ktu.edu.tr}

\section{Keywords:}

$900 \mathrm{Mhz}$ Electromagnetic field

Lipid peroxidation

Newborn rat testicle

Seminiferous tubules

\section{ABSTRACT}

Intensive use in daily life of devices with an electromagnetic field (EMF), such as radios, televisions, computers and mobile phones, results in constant exposure. The fact that more than $80 \%$ of people in many countries use mobile phones has further increased concerns about the effect of EMFs. The aim of this study was to investigate the effect of exposure to a 900-megahertz (MHz) EMF in the prenatal term on the newborn rat testicles. Six pregnant, female Sprague Dawley rats were equally divided into control (CG) and EMF (EMFG) groups. No procedure was performed on CG. EMFG was exposed to a $900 \mathrm{MHz}$ EMF for $1 \mathrm{~h}$ during days 13-21 of pregnancy at the same hour every day. No procedure was performed on newborn female rats after birth, and the study continued with newborn male rats. Newborn CG (NCG, n: 10) male rats were obtained from mothers in the CG, and newborn EMFG (NEMFG, n: 10) male rats from mothers in the EMFG. All animals were sacrificed and the testicles extracted at postnatal day 21 . Testicular tissue samples were evaluated histopathologically under light microscope. The TUNEL technique was used to determine apoptosis in testicles. Lipid peroxidation and DNA oxidation levels were evaluated biochemically. Histopathologically, NEMFG male rats exhibited irregularities in the seminiferous tubule basal membrane and epithelium of seminiferous tubules, immature germ cells in the lumen, and a decreased diameter of seminiferous tubules and thickness of epithelium. Apoptotic index, lipid peroxidation and DNA oxidation were higher in NEMFG male rats than in NCG. Our results showed that the newborn rat testicles exposed to $900 \mathrm{MHz}$ EMF in the prenatal period has been adversely affected.

J. Exp. Clin. Med., 2013; 30: 274 